\title{
Beating the Standard Sensitivity-Bandwidth Limit of Cavity-Enhanced Interferometers with Internal Squeezed-Light Generation
}

\author{
M. Korobko, ${ }^{1, *}$ L. Kleybolte, ${ }^{1}$ S. Ast,${ }^{2}$ H. Miao, ${ }^{3}$ Y. Chen, ${ }^{4}$ and R. Schnabel ${ }^{1}$ \\ ${ }^{1}$ Institut für Laserphysik und Zentrum für Optische Quantentechnologien, Universität Hamburg, \\ Luruper Chaussee 149, 22761 Hamburg, Germany \\ ${ }^{2}$ Institut für Gravitationsphysik, Leibniz Universität Hannover and Max-Planck-Institut für Gravitationsphysik \\ (Albert-Einstein-Institut), Callinstraße 38, 30167 Hannover, Germany \\ ${ }^{3}$ Institute of Gravitational Wave Astronomy, University of Birmingham, Birmingham B15 2TT, United Kingdom \\ ${ }^{4}$ Theoretical Astrophysics 350-17, California Institute of Technology, Pasadena, California 91125, USA
}

(Received 12 February 2017; published 7 April 2017)

\begin{abstract}
The shot-noise limited peak sensitivity of cavity-enhanced interferometric measurement devices, such as gravitational-wave detectors, can be improved by increasing the cavity finesse, even when comparing fixed intracavity light powers. For a fixed light power inside the detector, this comes at the price of a proportional reduction in the detection bandwidth. High sensitivity over a large span of signal frequencies, however, is essential for astronomical observations. It is possible to overcome this standard sensitivity-bandwidth limit using nonclassical correlations in the light field. Here, we investigate the internal squeezing approach, where the parametric amplification process creates a nonclassical correlation directly inside the interferometer cavity. We theoretically analyze the limits of the approach and measure $36 \%$ increase in the sensitivity-bandwidth product compared to the classical case. To our knowledge, this is the first experimental demonstration of an improvement in the sensitivity-bandwidth product using internal squeezing, opening the way for a new class of optomechanical force sensing devices.
\end{abstract}

DOI: 10.1103/PhysRevLett.118.143601

Introduction.-Optical cavities can be used to enhance the sensitivity of interferometric measurements of small signals caused by a weak classical force acting on a movable mirror. The motion of the mirror produces a phase modulation on the light field, which then gets enhanced by constructive interference with itself on the cavity round trip. For any given light power inside the detector cavity, increasing the cavity finesse improves the shot-noise limited sensitivity but is necessarily accompanied by a proportional reduction of the detection bandwidth [1,2]. This effect limits the performance of all gravitational-wave detectors (Advanced LIGO, GEO600, Advanced Virgo, KAGRA) [3-6]. Typical gravitational-wave signals require high but also broadband sensitivity: the signal from a binary black hole merger, such as the one detected in September 2015 [7], sweeps through the frequencies of the interferometer's detection band.

According to the Heisenberg uncertainty principle, one has to increase the uncertainty in the light's amplitude quadrature in order to improve the measurement sensitivity by decreasing the uncertainty in the light's phase quadrature. Since energy is needed to increase the uncertainty, the sensitivity limit of an interferometer is set by the optical energy inside the cavity $[8,9]$. In a more general case of arbitrary signal waveforms, this consideration leads to the quantum CramerRao bound (QCRB) for the estimation of signal in Gaussian quantum noise: at each signal frequency, the maximal phase sensitivity is set by the size of the amplitude quadrature uncertainty at the same frequency $[10,11]$.
Based on the QCRB, first of all, the concept of enhancing the sensitivity with an optical cavity can be understood. Both amplitude and phase quadratures resonate inside the cavity, and have their uncertainties amplified within the bandwidth of the resonance, and attenuated at other frequencies. In the case of a coherent input field and a simple Fabry-Perot cavity, the state remains coherent inside the cavity. The standard sensitivity-bandwidth limit is defined as the maximum product of a peak sensitivity $\mathcal{S}$ and a detection bandwidth $\mathcal{B}$, that can be achieved using coherent states of light and a given light power $P_{c}$ inside the cavity [12]: $\mathcal{S} \times \mathcal{B} \leq$ $8 \pi P_{c} /(\hbar \lambda L)$, where $\lambda$ is the optical wavelength, $L$ is the cavity length, and $\hbar$ is the reduced Planck constant.

We introduce a set of different strategies for improving the sensitivity of a cavity-enhanced interferometer beyond the standard sensitivity-bandwidth limit. The first approach is called the white-light cavity effect. It broadens the cavity resonance without changing the finesse, in which case the uncertainty of the amplitude quadrature must increase above the vacuum level. Recently, it was proposed that the white-light cavity effect can be achieved by using an anomalously dispersive medium inside the interferometer [13-22].

The second approach is called external squeezing. In this case, the uncertainty of the optical field injected in the interferometer is squeezed below the vacuum level in the phase quadrature, without influencing the signal enhancement due to the optical cavity [3,23-26]. The bandwidth remains unchanged, and hence, the standard 
sensitivity-bandwidth limit is surpassed due to the increased peak signal-to-noise ratio.

The third approach is internal squeezing [27-29]. Here, squeezed states of light are produced inside the detector's cavity, for instance, using an optical parametric amplifier. In contrast to external squeezing, in this approach, the phase quadrature squeezing happens mainly inside the optical cavity linewidth and affects both the noise and the signal. The amplitude quadrature uncertainty is correspondingly increased above the vacuum level, and in accordance with the QCRB, the sensitivity increases: the noise is squeezed more than the signal is deamplified. The detection bandwidth narrows in this case, but the peak sensitivity is increased even more strongly, which allows the standard sensitivity-bandwidth limit to be surpassed.

In this Letter, we analyze the third approach theoretically and report on a proof-of-principle experiment in which the standard sensitivity-bandwidth limit was surpassed by $36 \%$. We focus on surpassing this limit by considering shot noise alone for sensing mirror displacement, ignoring radiation-pressure noise. In practice, the radiation-pressure noise can either be suppressed by increasing the mass of mirrors [30], or evaded by using quantum nondemolition measurement techniques, e.g., frequency-dependent homodyne detection [8,31-33].

General concept.-We consider the propagation of a signal through a Fabry-Perot cavity with a nonlinear crystal inside, see Fig. 1. Pumping the crystal with light of the doubled frequency leads to optical parametric amplification of the cavity mode. The highest squeeze factor inside the cavity is achieved around cavity resonance and is limited to $6 \mathrm{~dB}$. At this level, the threshold for optical parametric oscillation is reached, and the amplified amplitude quadrature becomes unstable and causes lasing [34,35]. However, the squeeze factor outside the cavity is not fundamentally limited due to destructive interference between the incoming coherent field and outgoing squeezing [36]. On the other hand, the signal originates from the inside of the cavity, and does not experience such interference. Therefore, the deamplification in the signal remains limited to $6 \mathrm{~dB}$. The resulting difference between noise squeezing and signal deamplification constitutes the gain in the signal-to-noise ratio (SNR), which represents the sensitivity of the detector. On the other hand, the bandwidth gets reduced, as the internal squeezing increases the sensitivity only inside the cavity linewidth and leaves it unchanged outside. Despite this, the sensitivity-bandwidth product is enhanced, according to the QCRB, as we amplify the amplitude quadrature fluctuations inside the cavity.

We present a simplified treatment of a mathematical model of the system, leaving a rigorous treatment for the Supplemental Material [37]. We define three quantities that influence the cavity bandwidth: cavity decay rate through the coupling mirror, squeezing rate, and the round-trip optical loss rate, correspondingly

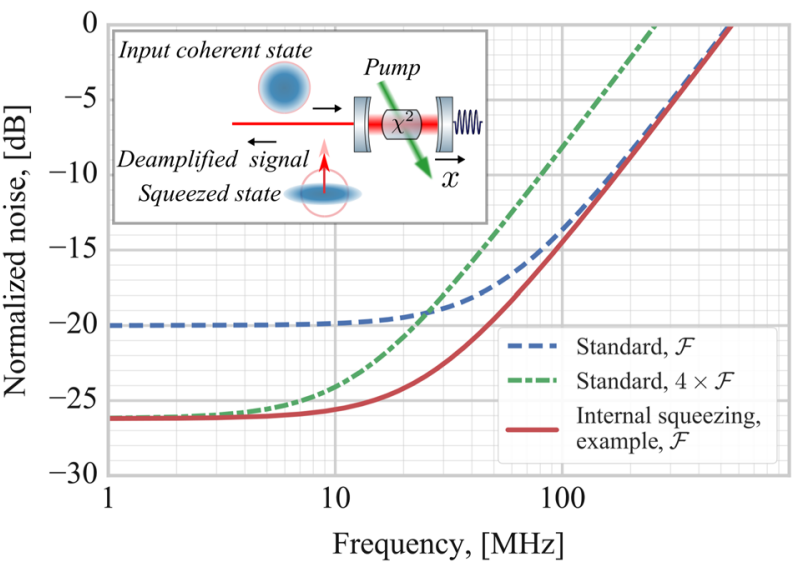

FIG. 1. The three curves show the quantum measurement noise of a cavity-enhanced interferometer with the same coherent light power in its arms, normalized to a phase signal optical transfer function. The peak sensitivity $\mathcal{S}$ is defined as inverse of the minimum of the curves; the bandwidth $\mathcal{B}$ is the frequency at which the noise rises by $3 \mathrm{~dB}$ above its minimal value. The standard sensitivity-bandwidth product remains constant for a given coherent light power inside the cavity: to increase the peak sensitivity by $6 \mathrm{~dB}$, the finesse $\mathcal{F}$ has to be increased by a factor of 4 , thus, the bandwidth decreases by the same amount (compare blue dashed and green dashed-dotted curves). The internal squeezing approach is depicted schematically on the subplot: a phase quadrature of the input coherent light field is squeezed inside the cavity, deamplifying the signal at the same time. Signal deamplification is less than the amount of squeezing on the detector, so the sensitivity increases. Increased amplitude quadrature, according to the QCRB, leads to enhancement of the sensitivity-bandwidth product beyond the standard limit. Therefore, when increasing the peak sensitivity by $6 \mathrm{~dB}$ of internal squeezing, the resulting bandwidth is broader (red solid curve) than the one achievable classically.

$$
\gamma_{c}=\frac{c t_{c}^{2}}{4 L}, \quad \gamma_{s}=\frac{q c}{L}, \quad \gamma_{l}=\frac{c l^{2}}{4 L},
$$

where $c$ is the speed of light, $L$ is the optical length of the cavity, $t_{c}$ is the amplitude transmissivity of the coupling mirror, $q$ is the squeeze factor on a single pass through a crystal, $l^{2}$ is the round-trip internal loss without the transmission of the coupling mirror.

From the optical fields' input-output relations we derive the power spectral density of noise of the output field detected by a balanced homodyne detector

$$
S_{n}(\Omega)=1-\frac{4 \gamma_{c} \gamma_{s} \eta}{\left(\gamma_{c}+\gamma_{l}+\gamma_{s}\right)^{2}+\Omega^{2}},
$$

where $\Omega$ is the signal frequency, $\eta$ is total detection efficiency including light propagation and the quantum efficiency of the homodyne. Correspondingly, for the optical transfer function $T(\Omega)$ of the phase modulation signal through the cavity to the detector, we find 


$$
|T(\Omega)|^{2} \approx \frac{8 \pi P_{c}}{\hbar \lambda L} \frac{\gamma_{c} \eta}{\left(\gamma_{c}+\gamma_{l}+\gamma_{s}\right)^{2}+\Omega^{2}} .
$$

The equations above lead to the definition of the common bandwidth for the noise and the signal transfer functions $\Gamma=\gamma_{c}+\gamma_{l}+\gamma_{s}$. Then, we define the sensitivity

$$
\frac{|T(\Omega)|^{2}}{S_{n}(\Omega)}=\frac{8 \pi P_{c}}{\hbar \lambda L} \frac{\gamma_{c} \eta}{\Gamma^{2}-4 \gamma_{c} \gamma_{s} \eta+\Omega^{2}} .
$$

Its peak value $\mathcal{S} \equiv|T(0)|^{2} / S_{n}(0)$ and bandwidth $\mathcal{B}$ are given by

$$
\begin{aligned}
\mathcal{S} & =\frac{8 \pi P_{c}}{\hbar \lambda L} \frac{\gamma_{c} \eta}{\mathcal{B}^{2}}, \\
\mathcal{B} & =\sqrt{\Gamma^{2}-4 \gamma_{c} \gamma_{s} \eta}
\end{aligned}
$$

From these equations, we can obtain an overall enhancement in the sensitivity-bandwidth product

$$
(\mathcal{S} \times \mathcal{B}) /(\mathcal{S} \times \mathcal{B})_{\gamma_{s}=0}=\frac{\gamma_{c}+\gamma_{l}}{\mathcal{B}} .
$$

For a given detection loss, there exists an optimal squeezing factor that gives maximal enhancement, which differs from the threshold value where the maximum squeezing is achieved. This can be understood as follows. The maximal detectable squeezing value is bounded by the amount of optical loss. The loss of squeezing can be seen as mixing with vacuum [41], therefore, above a certain value, the increase in squeezing is not detectable any more, see Eq. (2). However, the signal deamplification is independent of the detection loss, and has a weaker dependence on the internal loss, see Eq. (3). Therefore, increasing the internal gain above a certain level leads to a larger detected deamplification in the signal than suppression in the shot noise level.

We experimentally test the presence of an enhancement in the sensitivity-bandwidth product compared to the standard limit and show the influence of the detection loss on it.

Experiment.-In our proof-of-principle experiment, the signal is generated by injection of a phase modulated field from the back of the Fabry-Perot cavity with an optical parametric amplifier inside. In terms of signal detection and observation of the internal squeezing effect, this approach can be viewed as a one-to-one analogy to a detector with a movable end mirror sensitive to the external force. The advantage of our approach is that it allows signal generation in a broad frequency band, which is necessary to observe the change in the detection bandwidth.

The experimental setup, shown in Fig. 2, consists of a second harmonic generation cavity, producing $775 \mathrm{~nm}$ light for optical-parametric amplification of the longitudinal resonance at $1550 \mathrm{~nm}$ of our internal squeezing cavity, here,

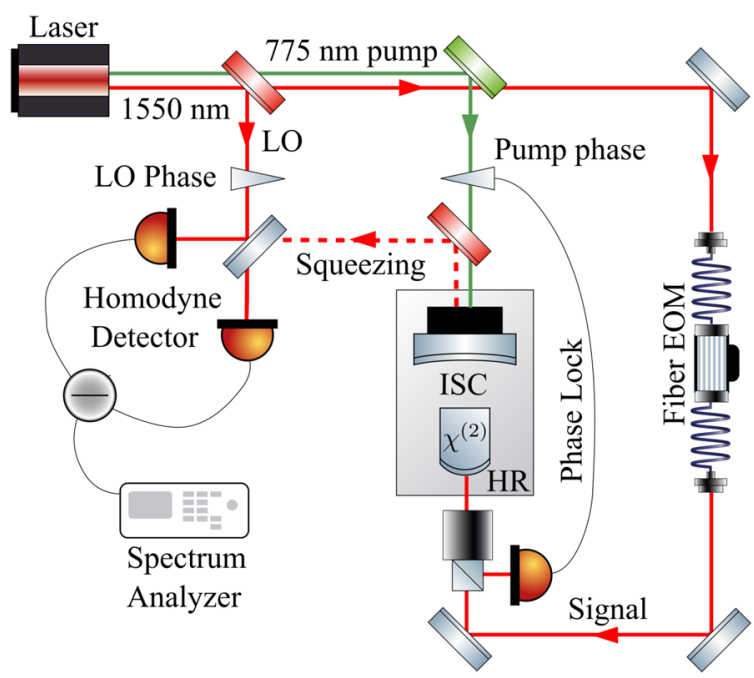

FIG. 2. Experimental setup-The ISC is resonant for both the fundamental wavelength $1550 \mathrm{~nm}$ and the second harmonic wavelength $775 \mathrm{~nm}$. Through the highly reflective (HR) back mirror, a beam at $1550 \mathrm{~nm}$ is injected carrying a phase modulation signal between 5.5 to $151 \mathrm{MHz}$ and a sideband at $54 \mathrm{MHz}$ for PDH cavity length stabilization. The output signal consisting of squeezed light and deamplified signal sideband is detected on a balanced homodyne detector using $2.8 \mathrm{~mW}$ local oscillator (LO) power, with an overall detection efficiency of $\sim 85 \%$. The phase of the local oscillator is actively stabilized to the phase quadrature, and the phase of the pump is stabilized to produce squeezing in the phase quadrature.

simply called "ISC." The cavity has an optical length of $L=2.77 \mathrm{~cm}$, an optical linewidth of $\gamma_{c} \sim 2 \pi \times 54 \mathrm{MHz}$, and contains a periodically poled KTP (PPKTP) crystal [42]. A control field at $1550 \mathrm{~nm}$ with a phase-modulation signal imprinted on it is injected from the highly reflective back side of the ISC. The signal is produced by the broadband fiber electro-optical modulator (EOM). The cavity length is stabilized via the Pound-Drever-Hall (PDH) locking technique $[43,44]$. The ISC has two locking modes-with and without the pump light. When the measurements with squeezing are taken, the cavity length is stabilized with $775 \mathrm{~nm}$ light, while the $1550 \mathrm{~nm}$ control field is used to stabilize the squeezing angle on the phase quadrature. When the measurements without squeezing are taken, the $775 \mathrm{~nm}$ pump is off, and the cavity length is stabilized with the $1550 \mathrm{~nm}$ control field. The signal with or without squeezing is detected with a highefficiency broadband homodyne detector with a bandwidth of $\sim 800 \mathrm{MHz}$ and dark noise clearance of $\sim 13 \mathrm{~dB}$ in the frequency range of interest from 10 to $200 \mathrm{MHz}$.

We create a phase modulation signal at different frequencies. At each frequency, we detect the signal together with the noise on the homodyne detector in two regimes: with the optical parametrical amplification being on and off. This allows us to observe how the signal gets deamplified, and noise-squeezed. From the squeezing spectrum we estimate the experimental parameters: squeezing factor $q$, transmissivity of the coupling mirror $t_{c}$, internal loss $l^{2}$, and 
detection efficiency $\eta$. The fitted upper bound on the internal loss of $l^{2} \leq 2300 \mathrm{ppm}$, which results in in the round-trip loss bandwidth of $\gamma_{l} \leq 2 \pi \times 743 \mathrm{kHz} \ll \gamma_{c}$, is consistent with the previously measured absorption of a PPKTP crystal [45], and the manufacturer specified transmissivity of the back mirror $\left(t_{b}^{2}=0.05 \% @ 1550 \mathrm{~nm}\right.$ ) and bound on the antireflective coating of the crystal $\left(r^{2}<0.1 \%\right)$. The coupling mirror transmissivity of $t_{c}^{2}=15 \% @ 1550 \mathrm{~nm}$ is confirmed by an independent measurement of the cavity finesse. The detection loss estimation is also bounded within $1 \%$ of the estimated value by comparing squeezing and antisqueezing spectra $[42,46]$. We use these estimated parameters to calculate the expected theoretical spectrum of the signal deamplification and compare it with the measured values.

Figure 3 compares noise squeezing with signal deamplification; the difference between the two data sets directly demonstrates the increase in the SNR, corresponding to an enhancement of $26 \%$ in the sensitivity-bandwidth product

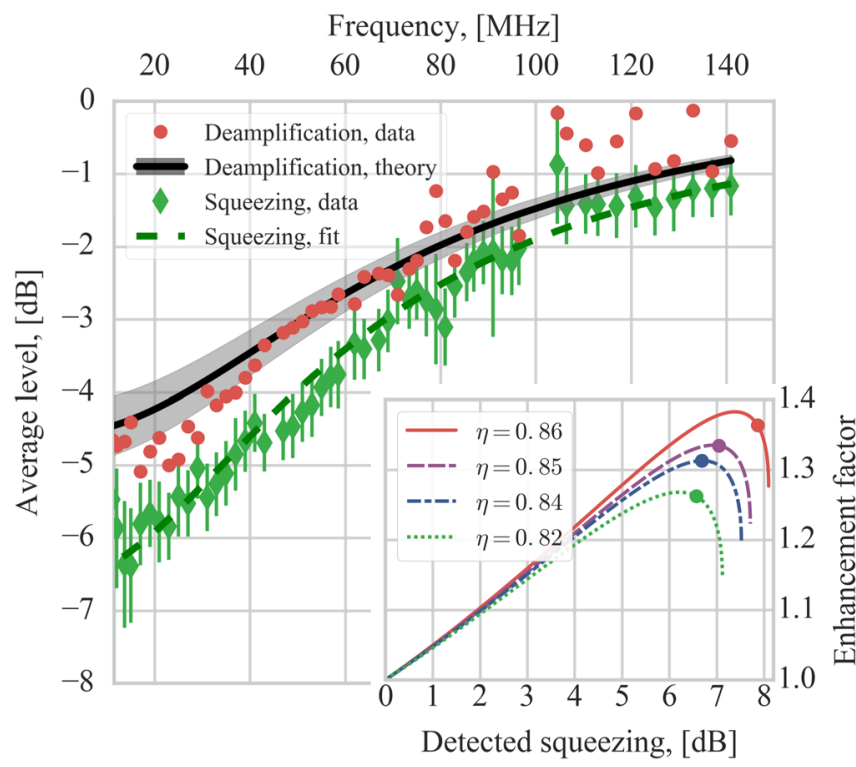

FIG. 3. Beating the standard sensitivity-bandwidth limit with internal squeezing. In the main plot, we demonstrate the increase in the signal-to-noise ratio for a total quantum efficiency of 0.82 : squeezing data (green rhomb) with fit (green dashed line), from which the squeezing factor is estimated; the signal deamplification is represented by the red dots. It is compared to the results of the theoretical modeling (black solid line) with parameters obtained from the squeezing measurement, where the grey area represents the confidence interval based on the estimation error. The subplot shows four experimentally achieved enhancement factors $(26 \%, 31 \%, 33 \%, 36 \%)$ representing four different overall quantum efficiencies, together with theoretical curves versus detected squeeze factor. Two effects are demonstrated: the dependence on the detection efficiency $\eta$ (different curves) and the existence of the optimal squeezing for each set of parameters. The maximal enhancement in the sensitivity-bandwidth product obtained in the experiment is $36 \%$ (red solid curve). The data on the main plot corresponds to the green (dotted) curve with $82 \%$ detection efficiency and $26 \%$ enhancement. beyond the standard limit. We find the theory to be in good agreement with the experimental data, lying within the confidence interval obtained from the parameter estimation error. We ascribe the observed discrepancies to the electronic resonances in the homodyne circuitry and wires that are not taken into account in the theoretical analysis. Higher enhancement factors are observed with a second homodyne detector, which has less loss (but also stronger electronic resonances). The dots in the subplot in Fig. 3 show four experimentally achieved enhancement factors $(26 \%, 31 \%$, $33 \%, 36 \%$ ) representing four different overall quantum efficiencies.

Summary and outlook.-In summary, we provide a unified view of three different nonclassical concepts for improving the quantum measurement noise limited sensitivity of cavity-enhanced laser interferometers. All of them can be seen as concepts of beating the standard sensitivitybandwidth limit. Two of these concepts: "white-light cavity" and "external squeezing," have been investigated intensively in recent years for the improvement of gravitational-wave detectors $[20,22,36]$. In this work, the third concept, "internal squeezing," is investigated, theoretically as well as experimentally and, also, in view of improving gravitational-wave detectors. We presented the first experimental demonstration of beating the standard sensitivity-bandwidth limit with internal squeezing. In application to gravitational-wave detectors, the nonlinear crystal would be placed in the dark output port, between the central beam splitter and the signalextraction mirror [47]. At this position, the low light power keeps thermally induced beam distortion as well as scattered light due to the crystal at a minimum.

We note that all three concepts can, in principle, be combined to maximize the overall improvement. The most mature concept is external squeezing, as it is already implemented in the gravitational-wave detector GEO 600 [3]. Since it avoids any deamplification of the signal and squeezes shot noise in a broadband way, it provides more improvement to the sensitivity-bandwidth product. Interestingly, its sensitivity to intracavity loss is higher than that of internal squeezing. This can be understood in the limiting case when the cavity round-trip loss equals the coupler's transmissivity. In this case, the cavity is impedance matched for external squeezing, and no squeezing gets reflected off the coupling mirror. By contrast, in the internal squeezing case, only half of the squeezing produced inside the cavity is lost. The other half is coupled out through the mirror, resulting in a maximal measurable squeeze factor of $3 \mathrm{~dB}$. Thus, based on our work, we propose to combine external and internal squeezing to improve the sensitivities of gravitational-wave detectors to values that are not possible with external squeezing alone.

We thank Sacha Kocsis for comments that greatly improved the manuscript, Paolo Piergentili for assistance with the electronics design, the members of MQM discussion group and LSC Quantum Noise working group for 
many fruitful discussions. The research of M.K. was supported by the Marie Curie Initial Training Network cQOM; the research of M. K., L. K., and R. S. is supported by the European Research Council (ERC) Project "MassQ" (Grant No. 339897); the research of S. A. was supported by the IMPRS on Gravitational-Wave Astronomy; the research of H.M. is supported by UK STFC Ernest Rutherford Fellowship; the research of R. S. is supported by Deutsche Forschungsgemeinschaft (Grant No. SCHN 757-6).

*mikhail.korobko@physnet.uni-hamburg.de

[1] C. M. Caves, Phys. Rev. D 26, 1817 (1982).

[2] A. A. Clerk, M. H. Devoret, S. M. Girvin, F. Marquardt, and R. J. Schoelkopf, Rev. Mod. Phys. 82, 1155 (2010).

[3] The LIGO Scientific Collaboration, Nat. Phys. 7, 962 (2011).

[4] Y. Aso, Y. Michimura, K. Somiya, M. Ando, O. Miyakawa, T. Sekiguchi, D. Tatsumi, and H. Yamamoto, Phys. Rev. D 88, 043007 (2013).

[5] The LIGO Scientific Collaboration, Classical Quantum Gravity 32, 074001 (2015).

[6] F. Acernese et al., Classical Quantum Gravity 32, 024001 (2015).

[7] The LIGO Scientific Collaboration, Phys. Rev. Lett. 116, 061102 (2016).

[8] V. B. Braginsky and F. Y. Khalili, Quantum Measurement (Cambridge University Press, Cambridge, England, 1992), Chap. V, p. 64.

[9] V. B. Braginsky, M. L. Gorodetsky, K. S. Thorne, and F. Y. Khalili, in Gravitational Waves, Third Edoardo Amaldi Conference, Pasadena, California, 2000, edited by S. Meshkov (American Institute of Physics, Melville, NY, 2000) pp. 180-189.

[10] M. Tsang, H. M. Wiseman, and C. M. Caves, Phys. Rev. Lett. 106, 090401 (2011).

[11] H. Miao, R. X. Adhikari, Y. Ma, B. Pang, and Y. Chen, arXiv:1608.00766.

[12] J. Mizuno, Ph.D thesis, Leibniz Universität Hannover 1995.

[13] A. Wicht, K. Danzmann, M. Fleischhauer, M. Scully, G. Müller, and R.-H. Rinkleff, Opt. Commun. 134, 431 (1997).

[14] A. Wicht, M. Müller, R. H. Rinkleff, A. Rocco, and K. Danzmann, Opt. Commun. 179, 107 (2000).

[15] S. Wise, G. Müller, D. Reitze, D. B. Tanner, and B. F. Whiting, Classical Quantum Gravity 21, S1031 (2004).

[16] S. Wise, V. Quetschke, A. J. Deshpande, G. Mueller, D. H. Reitze, D. B. Tanner, B. F. Whiting, Y. Chen, A. Tünnermann, E. Kley, and T. Clausnitzer, Phys. Rev. Lett. 95, 013901 (2005).

[17] G. S. Pati, M. Salit, K. Salit, and M. S. Shahriar, Phys. Rev. Lett. 99, 133601 (2007).

[18] H. N. Yum, J. Scheuer, M. Salit, P. R. Hemmer, and M. S. Shahriar, J. Lightwave Technol. 31, 3865 (2013).

[19] M. Zhou, Z. Zhou, and S. M. Shahriar, Phys. Rev. D 92, 082002 (2015).

[20] Y. Ma, H. Miao, C. Zhao, and Y. Chen, Phys. Rev. A 92, 023807 (2015).
[21] J. Qin, C. Zhao, Y. Ma, L. Ju, and D. G. Blair, Opt. Lett. 40, 2337 (2015).

[22] H. Miao, Y. Ma, C. Zhao, and Y. Chen, Phys. Rev. Lett. 115, 211104 (2015).

[23] C. M. Caves, Phys. Rev. Lett. 45, 75 (1980).

[24] C. M. Caves, Phys. Rev. D 23, 1693 (1981).

[25] R. Schnabel, N. Mavalvala, D. E. McClelland, and P. K. Lam, Nat. Commun. 1, 121 (2010).

[26] The LIGO Scientific Collaboration, Nat. Photonics 7, 613 (2013).

[27] H. Rehbein, J. Harms, R. Schnabel, and K. Danzmann, Phys. Rev. Lett. 95, 193001 (2005).

[28] V. Peano, H. G. L. Schwefel, C. Marquardt, and F. Marquardt, Phys. Rev. Lett. 115, 243603 (2015).

[29] K. Somiya, Y. Kataoka, J. Kato, N. Saito, and K. Yano, Phys. Lett. A 380, 521 (2016).

[30] B. P. Abbott et al., Classical Quantum Gravity 34, 044001 (2017).

[31] H. J. Kimble, Y. Levin, A. B. Matsko, K. S. Thorne, and S. P. Vyatchanin, Phys. Rev. D 65, 022002 (2001).

[32] F. Y. Khalili and S. L. Danilishin, in Progress in Optics, 1st ed. (Elsevier, New York, 2016) pp. 113-236.

[33] H. Miao, H. Yang, R. X Adhikari, and Y. Chen, Classical Quantum Gravity 31, 165010 (2014).

[34] G. Milburn and D. Walls, Opt. Commun. 39, 401 (1981).

[35] M. J. Collett and C. W. Gardiner, Phys. Rev. A 30, 1386 (1984).

[36] R. Schnabel, arXiv:1611.03986v1.

[37] See Supplemental Material at http://link.aps.org/ supplemental/10.1103/PhysRevLett.118.143601 for details of the calculation of the noise spectral density, signal transfer function, and sensitivity-bandwidth product for a FabryPerot cavity with losses, which includes Refs. [37-39].

[38] S. L. Danilishin and F. Y. Khalili, Living Rev. Relativ. 15, 5 (2012).

[39] C. M. Caves and B. L. Schumaker, Phys. Rev. A 31, 3068 (1985).

[40] B. L. Schumaker and C. M. Caves, Phys. Rev. A 31, 3093 (1985).

[41] G. Grynberg, A. Aspect, and C. Fabre, Introduction to Quantum Optics: From the Semi-Classical Approach to Quantized Light (Cambridge University Press, Cambridge, England, 2010), p. 696.

[42] M. Mehmet, S. Ast, T. Eberle, S. Steinlechner, H. Vahlbruch, and R. Schnabel, Opt. Express 19, 25763 (2011).

[43] R. W. P. Drever, J. L. Hall, F. V. Kowalski, J. Hough, G. M. Ford, A. J. Munley, and H. Ward, Appl. Phys. B 31, 97 (1983).

[44] E. D. Black, Am. J. Phys. 69, 79 (2001).

[45] J. Steinlechner, S. Ast, C. Krüger, A. Singh, T. Eberle, V. Händchen, and R. Schnabel, Sensors 13, 565 (2013).

[46] H. Vahlbruch, M. Mehmet, K. Danzmann, and R. Schnabel, Phys. Rev. Lett. 117, 110801 (2016).

[47] J. Mizuno, K. Strain, P. Nelson, J. Chen, R. Schilling, A. Rüdiger, W. Winkler, and K. Danzmann, Phys. Lett. A 175, 273 (1993). 